\title{
Simulation-based time evaluation of basic manual assembly tasks
}

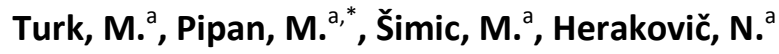 \\ ${ }^{a}$ University of Ljubljana, Faculty of Mechanical Engineering, Slovenia
}

\begin{abstract}
A B S T R A C T
The paper presents a simple simulation model of the lifting procedure that can be used to predict the total time required for the sequence of basic manual assembly tasks depending on the various parameters of the load and with regard to the workers' health. The aim of the research is to determine the appropriateness of using simulation tool for (re)setting time standards for manual assembly tasks. An avatar in the simulation model performs sequences of tasks with a handling mass of up to $20.5 \mathrm{~kg}$. The individual times obtained from the simulation model were analysed and compared with several time prediction methods and validated in laboratory environment. An analysis of the influence of different load parameters on the total time was also performed. Dependency is mostly linear, so from the practitioner point of view, we can predict with reasonable certainty the total time for any sequence of manual assembly tasks for every size and mass of the box. Based on the results we can confirm that simulation tool JACK is suitable not only for ergonomic analyses but also for setting time standards for the workers. Furthermore, with the simulation tool we analyse the process and get the accurate results in shorter time compared to other mentioned methods.
\end{abstract}

\section{ARTICLE INFO}

Keywords:

Assembly;

Manual task;

Work-job design;

Time analysis;

Jack simulation;

Avatar

*Corresponding author: miha.pipan@fs.uni-lj.si

(Pipan, M.)

Article history:

Received 20 April 2020

Revised 25 September 2020

Accepted 29 September 2020

\section{References}

[1] Nogueira, H.C., Locks, F., Barbieri, D.F., Oliveira, A.B. (2018). How does the biomechanical exposure of the upper body in manual box handling differ from exposure in other tasks in the real industrial context?, International Journal of Industrial Ergonomics, Vol. 68, 8-14, doi: 10.1016/j.ergon.2018.05.015.

[2] Borgs, S.P., La Delfa, N.J., Dickerson, C.R. (2019). An evaluation of off-axis manual forces and upper extremity joint moments during unilateral pushing and pulling exertions, Ergonomics, Vol. 62, No. 1, 52-64, doi: 10.1080/00140139.2018.1525501.

[3] Leber, M., Bastič, M., Moody, L., Schmidt Krajnc, M. (2018). A study of the impact of ergonomically designed workplaces on employee productivity, Advances in Production Engineering \& Management, Vol 13, No. 1, 107 117, doi: 10.14743/apem2018.1.277.

[4] Rasmussen, C.D.N., Højberg, H., Bengtsen, E., Jørgensen, M.B. (2018). Identifying knowledge gaps between practice and research for implementation components of sustainable interventions to improve the working environment - A rapid review, Applied Ergonomics, Vol. 67, 178-192, doi: 10.1016/i.apergo.2017.09.014.

[5] Dianat, I., Molenbroek, J., Castellucci, H.I. (2018). A review of the methodology and applications of anthropometry in ergonomics and product design, Ergonomics, Vol. 61, No. 12, 1696-1720, doi: 10.1080/00140139. 2018.1502817.

[6] de Mattos, D.L., Ariente Neto, R., Merino, E.A.D, Forcellini, F.A. (2019). Simulating the influence of physical overload on assembly line performance: A case study in an automotive electrical component plant, Applied Ergonomics, Vol. 79, 107-121, doi: 10.1016/i.apergo.2018.08.001.

[7] Lanzotti, A., Vanacore, A., Tarallo, A., Nathan-Roberts, D., Coccorese, D., Minopoli, V., Carbone, F., d'Angelo, R., Grasso, C., Di Gironimo, G., Papa, S. (2019). Interactive tools for safety 4.0: Virtual ergonomics and serious games in real working contexts, Ergonomics, Vol. 63, No. 3, 324-333, doi: 10.1080/00140139.2019.1683603. 
[8] Harari, Y., Bechar, A., Raschke, U., Riemer, R. (2017). Automated simulation-based workplace design that considers ergonomics and productivity, International Journal of Simulation Modelling, Vol. 16, No. 1, 5-18, doi: 10.2507 /IJSIMM16(1)1.355.

[9] Harari, Y., Riemer, R., Bechar, A. (2018). Factors determining workers' pace while conducting continuous sequential lifting, carrying, and lowering tasks, Applied Ergonomics, Vol. 67, 61-70, doi: 10.1016/i.apergo.2017. $\underline{09.003}$.

[10] Gupta, I., Kalra, P., Chawla, P., Singh, J. (2018). Evaluation of pilot's seat design of civil aircraft for Indian anthropometric data by using Delmia human software, Procedia Manufacturing, Vol. 26, 70-75, doi: 10.1016/ j.promfg.2018.07.009.

[11] Padula, R.S., Coury, H.J.C.G. (2003). Sagittal trunk movements during load carrying activities: A pilot study, International Journal of Industrial Ergonomics, Vol. 32, No. 3, 181-188, doi: 10.1016/S0169-8141(03)00062-3.

[12] Martinez, R., Bouffard, J., Michaud, B., Plamondon, A., Côté, J.N., Begon, M. (2019). Sex differences in upper limb 3D joint contributions during a lifting task, Ergonomics, Vol. 62, No. 5, 682-693, doi: 10.1080/00140139. 2019.1571245.

[13] Corbeil, P., Plamondon, A., Handrigan, G., Vallée-Marcotte, J., Laurendeau, S., Ten Have, J., Manzerolle, N. (2019). Biomechanical analysis of manual material handling movement in healthy weight and obese workers, Applied Ergonomics, Vol. 74, 124-133, doi: 10.1016/j.apergo.2018.08.018.

[14] Parida, R., Ray, P.K. (2015). Biomechanical modelling of manual material handling tasks: A comprehensive review, Procedia Manufacturing, Vol. 3, 4598-4605, doi: 10.1016/j.promfg.2015.07.539.

[15] Plamondon, A., Larivière, C., Denis, D., Mecheri, H., Nastasia, I. (2017). Difference between male and female workers lifting the same relative load when palletizing boxes, Applied Ergonomics, Vol. 60, 93-102, doi: 10.1016/ j.apergo.2016.10.014.

[16] Theurel, J., Desbrosses, K., Roux, T., Savescu, A. (2018). Physiological consequences of using an upper limb exoskeleton during manual handling tasks, Applied Ergonomics, Vol. 67, 211-217, doi: 10.1016/j.apergo.2017. $\underline{10.008}$.

[17] Pinder, A.D.J., Boocock, M.G. (2014). Prediction of the maximum acceptable weight of lift from the frequency of lift, International Journal of Industrial Ergonomics, Vol. 44, No. 2, 225-237, doi: 10.1016/j.ergon.2012.11.005.

[18] Lee, T.-H. (2003). Minimal acceptable handling time intervals for lifting and lowering tasks, Applied Ergonomics, Vol. 34, No. 6, 629-634, doi: 10.1016/S0003-6870(03)00050-4.

[19] Straker, L.M., Stevenson, M.G., Twomey, L.T. (1996). A comparison of risk assessment of single and combination manual handling tasks: 1. Maximum acceptable weight measures, Ergonomics, Vol. 39, No. 1, 128-140, doi: $10.1080 / 00140139608964439$.

[19] Straker, L.M., Stevenson, M.G., Twomey, L.T., Smith, L.M. (1997). A comparison of the risk assessment of single and combined manual handling tasks: 3. Biomechanical measures, Ergonomics, Vol. 40, No. 7, 708-728, doi: $10.1080 / 001401397187856$.

[21] Ahmed, S., Gawand, M.S., Irshad, L., Demirel, H.O. (2018). Exploring the design space using a surrogate model approach with digital human modelling simulation, In: Proceedings of the ASME 2018 International Design Engineering Technical Conferences and Computers and Information in Engineering Conference, Volume 1B: 38th Computers and Information in Engineering Conference, Quebec, Canada, 1-13, doi: 10.1115/DETC2018-86323.

[22] Vujica Herzog, N., Harih, G. (2019). Decision support system for designing and assigning ergonomic workplaces to workers with disabilities, Ergonomics, Vol. 63, No. 2, 225-236, doi: 10.1080/00140139.2019.1686658.

[23] Harari, Y., Bechar, A., Riemer, R. (2020). Workers' biomechanical loads and kinematics during multiple-task manual material handling, Applied Ergonomics, Vol. 83, Article No. 102985, doi: 10.1016/j.apergo.2019.102985.

[24] Harari, Y., Bechar, A., Riemer, R. (2019). Simulation-based optimization methodology for a manual material handling task design that maximizes productivity while considering ergonomic constraints, IEEE Transactions on Human-Machine Systems, Vol. 49, No. 5, 440-448, doi: 10.1109/THMS.2019.2900294.

[25] Vujica Herzog, N., Buchmeister, B., Beharic, A., Gajsek, B. (2018). Visual and optometric issues with smart glasses in Industry 4.0 working environment, Advances in Production Engineering \& Management, Vol. 13, No. 4, 417428, doi: 10.14743/apem2018.4.300.

[26] Klodawski, M., Jachimowski, R., Jacyna-Golda, I., Izdebski, M. (2018). Simulation analysis of order picking efficiency with congestion situations, International Journal of Simulation Modelling, Vol. 17, No. 3, 431-443, doi: 10.2507/IJSIMM17(3)438.

[27] Turk, M., Resman, M., Herakovič, N. (2018). Preparation of papers for IFAC conferences \& symposia: Computeraided processing of manual assembly operations with integration of simulation tools in production processes, IFAC-PapersOnLine, Vol. 51, No. 2, 813-818, doi: 10.1016/j.ifacol.2018.04.014.

[28] Karger, D.W., Bayha, F.H. (1961). Engineered Work Measurement, First edition, Industrial Press, New York, USA. 


\title{
Simulacijsko podprto vrednotenje časa izvedbe osnovnih nalog pri ročni montaži
}

\author{
Turk, M. ${ }^{a}$, Pipan, M. ${ }^{\mathrm{a},{ }^{*},}$ Šimic, M. ${ }^{a}$, Herakovič, N. ${ }^{\mathrm{a}}$ \\ aUniversity of Ljubljana, Faculty of Mechanical Engineering, Slovenia
}

\section{POVZETEK}

$\mathrm{V}$ prispevku je predstavljen preprost simulacijski model postopka dvigovanja $\mathrm{S}$ pomočjo simulacijskega modela je mogoče predvideti skupni čas, potreben za izvedbo zaporedja osnovnih nalog pri ročni montaži, v odvisnosti od različnih parametrov obremenitve in ob upoštevanju zdravja delavcev. Cilj raziskave je ugotoviti primernost uporabe simulacijskega orodja za določanje (oz. ponovno določanje) časovnih standardov za ročno montažo. Avatar v simulacijskem modelu izvaja zaporedja nalog, pri katerih upravlja predmete z maso do $20,5 \mathrm{~kg}$. Posamezne čase, pridobljene s simulacijskim modelom, smo analizirali in primerjali z več metodami časovnega napovedovanja ter potrdili $\mathrm{v}$ laboratorijskem okolju. Opravljena je bila tudi analiza vpliva različnih parametrov obremenitve na skupni čas. Odvisnost je večinoma linearna, zato lahko $\mathrm{z}$ vidika delavca z razumno gotovostjo predvidimo skupni čas za izvedbo katerega koli zaporedja nalog ročne montaže za vsako velikost in maso škatle. Na podlagi rezultatov lahko potrdimo, da je simulacijsko orodje JACK primerno ne samo za ergonomske analize, temveč tudi za določanje časovnih standardov za delavce. Poleg tega lahko s simulacijskim orodjem analiziramo postopek in dobimo natančne rezultate $\mathrm{v}$ krajšem času v primerjavi z drugimi omenjenimi metodami.

\section{PODATKI O ČLANKU}

Ključne besede:

Montaža;

Ročna naloga;

Načrtovanje izvedbe dela;

Analiza časa;

Simulacija Jack;

Avatar

* Kontaktna oseba:

miha.pipan@fs.uni-lj.si

(Pipan, M.)

Zgodovina članka:

Prejet 20. aprila 2020

Popravljen 25. septembra 2020

Sprejet 29. septembra 2020 